\title{
Manejo de la diverticulitis yeyunal. Experiencia en nuestro centro
}

\author{
Management of jejunal diverticulitis. Experience in our center \\ Javier Serrano-González*, Mariano Artés-Caselles, Laura Román-García de León, Pau Plá-Sánchez y \\ Víctor Sánchez-Turrión \\ Servicio de Cirugía General y del Aparato Digestivo, Hospital Universitario Puerta de Hierro Majadahonda, Majadahonda, Madrid, España
}

\begin{abstract}
Resumen
La enfermedad diverticular yeyunal es una condición clínica muy poco frecuente y habitualmente asintomática. Las complicaciones asociadas aparecen en menos del 30\% de los pacientes y pueden manifestarse como inflamación de los divertículos, obstrucción intestinal, hemorragia digestiva, malabsorción intestinal, formación de abscesos intraabdominales y perforación. La clínica de los pacientes es muchas veces inespecífica, requiriendo un alto grado de sospecha para llegar al diagnóstico. El tratamiento de las complicaciones de la enfermedad diverticular es variado, incluyendo reposición de volumen o transfusiones, antibioticoterapia, drenaje percutáneo o intervención quirúrgica. Presentamos un estudio observacional retrospectivo de los casos tratados en nuestro hospital entre 2007 y 2016.
\end{abstract}

PALABRAS CLAVE: Yeyuno. Diverticulosis. Diverticulitis. Perforación intestinal. Resección intestinal.

\begin{abstract}
Jejunal diverticular disease is a very uncommon pathology often asymptomatic. Associated complications appear in less than $30 \%$ of patients and they can present as diverticulitis, refractary inflammation, obstruction, hemorrhage, perforation or intraabdominal abscess formation. Clinical manifestations are usually unspecific and high suspicion index is required to reach the diagnosis. Treatment of complications includes volume replacement, transfusions, antibiotic therapy, percutaneous drainage or surgical intervention. We present a retrospective observational study of the cases treated in our hospital between 2007 and 2016.
\end{abstract}

KEY WORDS: Jejunal. Diverticulosis. Diverticulitis. Intestinal perforation. Intestinal resection.

\section{Correspondencia:}

*Javier Serrano González

Manuel de Falla, 1

28222 Majadahonda (Madrid), España

E-mail: javierserranogdr@gmail.com
Fecha de recepción: 18-12-2017

Fecha de aceptación: 11-04-2018

DOI://dx.doi.org/10.24875/CIRU.M18000024
Cir Cir. 2018;86:148-151

Contents available at PubMed www.cirugiaycirujanos.com 


\section{Introducción}

Los divertículos son formaciones saculares formadas a partir de la pared intestinal. Son mucho más frecuentes en el colon, aunque también pueden encontrarse en el intestino delgado; de los que aparecen en el intestino delgado, los duodenales tienen una mayor prevalencia $(80 \%)$ respecto a los localizados en el yeyuno y el íleon (20\%)1. Según las capas de la pared que estén afectas, se pueden clasificar en divertículos verdaderos (afectando a toda la pared intestinal) y pseudodivertículos (en los que se hernian la mucosa y la submucosa a través de la capa muscular, habitualmente en puntos de debilidad de la pared, coincidiendo con el paso de los vasos nutricios a través del meso) $)^{2,3}$, siendo más frecuentes estos últimos. También pueden dividirse en intraluminales (congénitos) o extraluminales (suelen ser adquiridos).

La enfermedad diverticular yeyunal es una condición clínica muy poco frecuente. La mayoría de los pacientes no sufren ningún síntoma asociado a esta enfermedad si no se producen complicaciones, las cuales sólo aparecen en un $10-30 \%$ de los casos. Estas complicaciones consisten en inflamación de los divertículos (diverticulitis), obstrucción intestinal por volvulación, sangrado, malabsorción intestinal, formación de abscesos intraabdominales y perforación libre a la cavidad abdominal|4-6. En estos casos, la clínica con la que pueden presentarse es muchas veces inespecífica, por lo que se requiere un alto grado de sospecha para su diagnóstico.

El diagnóstico es, en la mayoría de las ocasiones, casual, en el curso de una prueba complementaria por otro motivo.

El tratamiento de los divertículos asintomáticos no está indicado, y sólo se realiza manejo de las complicaciones en caso de que se estas se produzcan?.

\section{Métodos}

Presentamos los resultados de un estudio retrospectivo observacional descriptivo realizado en nuestro hospital, centro de tercer nivel, de pacientes diagnosticados de diverticulitis yeyunal entre $2007 \mathrm{y}$ 2016. Todos los pacientes fueron diagnosticados mediante tomografía computarizada (TC) abdominal. Se analizaron en total 12 casos. Los datos recogidos incluyeron edad de presentación del cuadro, sexo, factores de riesgo de mala evolución, presencia de datos de complicación, tratamiento realizado y posterior evolución de cada paciente.
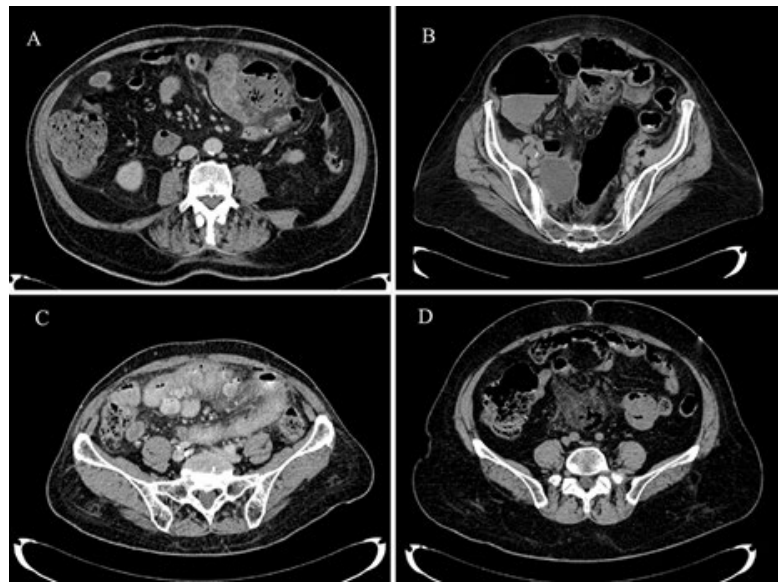

Figura 1. A: engrosamiento de asa yeyunal con cambios inflamatorios de asa y grasa adyacente sin signos de complicación. B: asa yeyunal con trabeculación de la grasa mesentérica y burbujas aisladas de neumoperitoneo. C: divertículo yeyunal con trabeculación de grasa adyacente y líquido libre. D: divertículo en pared de unión yeyuno-ileal, con inflamación en raíz del meso, y burbujas de gas contenidas por grasa mesentérica adyacente.

\section{Resultados}

Se han analizado 12 pacientes, 7 hombres (58\%) y 5 mujeres (42\%), con una edad media de 79 años (rango: 69-92 años). Entre la comorbilidad que presentaban destacan un paciente en tratamiento quimioterápico por una leucemia mieloide aguda, un paciente con insuficiencia renal crónica, tres pacientes con cuadro de demencia y cinco con hipertensión arterial en tratamiento. Cuatro de nuestros pacientes habían tenido cirugías abdominales previas.

Todos los pacientes estudiados presentaron dolor abdominal como motivo de consulta, apareciendo fiebre o vómitos, o ambos, sólo en cuatro casos (33\%). La mitad de los pacientes presentaron leucocitosis en la analítica realizada en urgencias, con un caso de neutrofilia sin leucocitosis.

En seis (50\%) de estos pacientes se encontraron datos en la TC abdominal de neumoperitoneo, mientras que en un caso (8\%) se objetivaron datos de obstrucción intestinal y en otro una colección abscesificada durante el ingreso. El resto de los pacientes presentaba únicamente inflamación de formaciones saculares dependientes del yeyuno (Fig. 1).

Dos pacientes fueron intervenidos de urgencia en el momento de llegar al hospital, realizándose en uno de ellos resección intestinal del segmento afecto (divertículo perforado previo a brida intestinal que condicionaba obstrucción retrógrada) y en el otro lavado de la cavidad abdominal y colocación de drenaje mediante abordaje laparoscópico, sin resección intestinal. Otro caso se intervino tras mala evolución con tratamiento antibiótico, con resección del área afecta 
Tabla 1. Resumen de los resultados de nuestra serie

\begin{tabular}{|c|c|c|c|c|c|c|c|c|}
\hline Sexo & Edad & $\begin{array}{l}\text { Cirugías } \\
\text { previas }\end{array}$ & $\begin{array}{l}\text { Clínica al } \\
\text { ingreso }\end{array}$ & Hallazgos en TC & Tratamiento & Evolución & Días de ingreso & Recurrencia \\
\hline Masculino & 88 & Sí & $\begin{array}{l}\text { Dolor } \\
\text { abdominal } \\
\text { Neutrofilia }\end{array}$ & $\begin{array}{l}\text { Inflamación } \\
\text { divertículos } \\
\text { yeyunales }\end{array}$ & $\begin{array}{l}\text { Médico } \\
\text { (ertapenem) }\end{array}$ & $\begin{array}{l}\text { Sin } \\
\text { complicaciones }\end{array}$ & 3 & No \\
\hline Masculino & 84 & No & $\begin{array}{l}\text { Dolor } \\
\text { abdominal }\end{array}$ & $\begin{array}{l}\text { Neumoperitoneo } \\
\text { sin líquido libre }\end{array}$ & $\begin{array}{l}\text { Médico } \\
\text { (ertapenem. AAC) }\end{array}$ & $\begin{array}{l}\text { Sin } \\
\text { complicaciones }\end{array}$ & 5 & No \\
\hline Masculino & 72 & No & $\begin{array}{l}\text { Dolor } \\
\text { abdominal } \\
\text { Fiebre } \\
\text { Vómitos } \\
\text { Leucocitosis }\end{array}$ & $\begin{array}{l}\text { Inflamación } \\
\text { divertículos } \\
\text { yeyunales } \\
\text { Divertículos en } \\
\text { duodeno y colon } \\
\text { no complicados }\end{array}$ & $\begin{array}{l}\text { Médico } \\
\text { (meropenem) }\end{array}$ & $\begin{array}{l}\text { Sin } \\
\text { complicaciones }\end{array}$ & 9 & No \\
\hline Masculino & 87 & Sí & $\begin{array}{l}\text { Dolor } \\
\text { abdominal } \\
\text { Leucocitosis }\end{array}$ & $\begin{array}{l}\text { Inflamación } \\
\text { divertículos } \\
\text { yeyunales con } \\
\text { plastrón asociado }\end{array}$ & $\begin{array}{l}\text { Médico } \\
\text { (PTZ, } \\
\text { ertapenem+ciprofloxacino) }\end{array}$ & Recidiva & 8 & Sí \\
\hline Femenino & 69 & No & $\begin{array}{l}\text { Dolor } \\
\text { abdominal } \\
\text { Fiebre } \\
\text { Leucocitosis }\end{array}$ & $\begin{array}{l}\text { Perforación } \\
\text { contenida, con } \\
\text { neumoperitoneo } \\
\text { en raíz del meso }\end{array}$ & $\begin{array}{l}\text { Médico } \\
\text { (PTZ) }\end{array}$ & $\begin{array}{l}\text { Sin } \\
\text { complicaciones }\end{array}$ & 8 & No \\
\hline Femenino & 76 & No & $\begin{array}{l}\text { Dolor } \\
\text { abdominal } \\
\text { Leucocitosis }\end{array}$ & $\begin{array}{l}\text { Divertículos } \\
\text { en yeyuno con } \\
\text { inflamación en } \\
\text { pared y meso }\end{array}$ & $\begin{array}{l}\text { Cirugía (resección intestinal } \\
\text { y anastomosis) tras } \\
\text { tratamiento médico } \\
\text { (PTZ) }\end{array}$ & $\begin{array}{l}\text { Mala evolución } \\
\text { con tratamiento } \\
\text { médico } \\
\text { Sin } \\
\text { complicaciones } \\
\text { posquirúrgicas }\end{array}$ & 21 & No \\
\hline Femenino & 78 & No & $\begin{array}{l}\text { Dolor } \\
\text { abdominal } \\
\text { Vómitos } \\
\text { Leucocitosis }\end{array}$ & $\begin{array}{l}\text { Inflamación } \\
\text { divertículos } \\
\text { yeyunales }\end{array}$ & $\begin{array}{l}\text { Drenaje percutáneo } \\
\text { (PTZ) }\end{array}$ & Reingreso & 60 & Recidiva \\
\hline Femenino & 78 & No & $\begin{array}{l}\text { Dolor } \\
\text { abdominal } \\
\text { Vómitos } \\
\text { Leucocitosis }\end{array}$ & $\begin{array}{l}\text { Trabeculación de } \\
\text { meso y burbujas } \\
\text { aisladas de } \\
\text { neumoperitoneo }\end{array}$ & $\begin{array}{l}\text { Médico } \\
\text { (ertapenem) }\end{array}$ & $\begin{array}{l}\text { Sin } \\
\text { complicaciones }\end{array}$ & 10 & No \\
\hline Masculino & 73 & Sí & $\begin{array}{l}\text { Dolor } \\
\text { abdominal }\end{array}$ & $\begin{array}{l}\text { Engrosamiento } \\
\text { pared yeyunal, } \\
\text { neumoperitoneo } \\
\text { Neumatosis portal }\end{array}$ & $\begin{array}{l}\text { Cirugía (lavado de cavidad } \\
\text { y drenaje) } \\
\text { Meropenem, } \\
\text { AAC }\end{array}$ & $\begin{array}{l}\text { Infección de } \\
\text { herida }\end{array}$ & 8 & No \\
\hline Masculino & 78 & No & $\begin{array}{l}\text { Dolor } \\
\text { abdominal } \\
\text { Fiebre }\end{array}$ & $\begin{array}{l}\text { Inflamación } \\
\text { divertículos } \\
\text { yeyunales }\end{array}$ & $\begin{array}{l}\text { Médico } \\
\text { (meropenem+voriconazol) }\end{array}$ & $\begin{array}{l}\text { Sin } \\
\text { complicaciones }\end{array}$ & 10 & No \\
\hline Masculino & 72 & No & $\begin{array}{l}\text { Dolor } \\
\text { abdominal } \\
\text { Fiebre }\end{array}$ & $\begin{array}{l}\text { Inflamación } \\
\text { divertículos } \\
\text { yeyunales }\end{array}$ & $\begin{array}{l}\text { Médico } \\
\text { (ertapenem) }\end{array}$ & $\begin{array}{l}\text { Sin } \\
\text { complicaciones }\end{array}$ & 6 & No \\
\hline Femenino & 92 & Sí & $\begin{array}{l}\text { Dolor } \\
\text { abdominal } \\
\text { Vómitos }\end{array}$ & $\begin{array}{l}\text { Signos obstrucción } \\
\text { intestinal } \\
\text { Neumoperitoneo }\end{array}$ & $\begin{array}{l}\text { Cirugía } \\
\text { Resección y anastomosis } \\
\text { Médico (AAC, PTZ) }\end{array}$ & $\begin{array}{l}\text { Sin } \\
\text { complicaciones }\end{array}$ & 10 & No \\
\hline
\end{tabular}

AAC: amoxicilina-ácido clavulánico; PTZ: piperacilina-tazobactam; TC: tomografía computarizada.

y anastomosis en un tiempo. La anatomía patológica en los dos casos en los que se realizó resección intestinal reveló datos de diverticulosis perforada con diverticulitis y peritonitis. Nueve pacientes (75\%) se trataron de forma conservadora con antibioticoterapia, requiriendo uno de ellos drenaje percutáneo de una colección intraabdominal.

El periodo de ingreso tuvo una media de 13 días, con un rango que osciló entre los 3 y los 60 días (este ingreso más prolongado corresponde a la paciente 
operada tras el fracaso del manejo conservador). El tratamiento antibiótico, que se mantuvo de forma ambulatoria en 10 de los 12 casos, se prolongó una media de 20 días (rango: 10-48).

Uno de los pacientes, tratado inicialmente de forma conservadora por hallazgos en la TC de diverticulitis yeyunal no complicada, precisó reingreso por mala evolución en su domicilio con antibioticoterapia vía oral. A su llegada a urgencias, el cuadro había evolucionado respecto al momento del alta, presentando una colección intraabdominal. Para su manejo se asociaron antibioticoterapia intravenosa y drenaje percutáneo de la colección.

En otro paciente se produjo una recidiva tardía, a los 2 años, sin aparición de complicaciones en ninguno de los dos ingresos y resolución de ambos con antibioticoterapia intravenosa.

No se registró mortalidad asociada a esta patología en nuestra serie (Tabla 1).

\section{Discusión}

La enfermedad diverticular de intestino delgado es poco frecuente, con una incidencia del $0.06-1.5 \%$ (hasta el $5 \%$ según series en autopsias) ${ }^{8}$, máxima a partir de la séptima década de la vida 9 . Sin embargo, la incidencia podría ser mayor, debido a que la mayoría de los pacientes permanecen asintomáticos. Los divertículos localizados en el yeyuno y el íleon son más frecuentes en los hombres. Suelen estar asociados a la existencia previa de trastornos en la motilidad intestinal, debidos a esclerosis sistémica progresiva, neuropatías y miopatías, y a la presencia de bridas posquirúrgicas que condicionan cuadros suboclusivos de repetición ${ }^{10}$. En la mayoría de los casos no aparecen complicaciones, pero puede ser causa de abdomen agudo.

La clínica puede ser muy inespecífica, con dolor abdominal difuso, malabsorción intestinal con diarreas y pérdida de peso, hemorragia digestiva baja, fiebre e, incluso, cuadro de abdomen agudo en caso de que se produzca la inflamación con o sin perforación de un divertículo ${ }^{11,12}$.

En los casos de complicación y cuadro agudo de dolor, fiebre, obstrucción intestinal, hematoquecia, pérdida de peso u otros síntomas, las pruebas a realizar son TC abdominal con contraste intravenoso, resonancia magnética 0 endoscopia ${ }^{13-15}$, o ambas. En ocasiones, las pruebas complementarias no son suficientes para llegar a un diagnóstico seguro, y se hace necesaria la exploración quirúrgica (laparotomía o laparoscopia).

Las complicaciones que pueden aparecer son inflamación, sangrado, obstrucción, formación de absceso y perforación de uno o varios divertículos ${ }^{16}$. Su manejo puede ser conservador, con maniobras de resucitación en el caso del sangrado y antibioticoterapia intravenosa cuando aparecen inflamación o absceso ${ }^{17,18}$. En caso de no responder a estas medidas, se requieren maniobras más agresivas, drenaje percutáneo o laparoscopia/laparotomía exploradora para resección intestinal del segmento afecto, seguida habitualmente de anastomosis primaria ${ }^{19-21}$.

\section{Agradecimientos}

Especial agradecimiento por su ayuda al impulso y la mejora del artículo a los doctores Javier López Monclús y José Luis Lucena de la Poza.

\section{Conflicto de intereses}

Todos los autores declaran no tener conflictos de intereses.

\section{Bibliografía}

1. Akhrass R, Yaffe MB, Fischer C, Ponsky J, Shuck JM. Small-bowel diverticulosis: perceptions and reality. J Am Coll Surg. 1997;184:383-8.

2. Makris K, Tsiotos GG, Stafyla V, Sakorafas GH. Small intestinal nonmeckelian diverticulosis: clinical review. J Clin Gastroenterol. 2009;43:201-7.

3. Chow DC, Babain M, Taubin HL. Jejunoileal diverticula. Gastroenterologist. 1997;5:78-84.

4. Kouraklis G, Mantas D, Glivanou A, Kouskos E, Raftopoulos J, Karatzas G. Diverticular disease of the small bowel: report of 27 cases. Int Surg.2001;86:235-9.

5. Doland JW. Major complications of small bowel diverticula. Ann Surg. 1979;190:183-88.

6. Kassir R, Boueil-Bourlier A, Baccot S. Jejuno-ileal diverticulitis: etiopathogenicity, diagnosis and management. Int J Surg Case Rep. 2015;10:151-3.

7. Singal R, Gupta S, Airon A. Giant and multiple jejunal diverticula presenting as peritonitis a significant challenging disorder. J Med Life. 2012;15:308-10.

8. Miller RE, McCabe RE, Salomon PF, Knox WG. Surgical complications of small bowel diverticula exclusive of Meckel's. Ann Surg. 1970;171:202-10.

9. De Bree E, Grammatikakis J, Christodoulakis M, Tsiftsis D. The clinical significance of acquired jejunoileal diverticula. Am J Gastroenterol. 1998; 93:2523-8.

10. Krishnamurthy S, Kelly MM, Rohrmann CA, Schuffler MD. Jejunal diverticulosis. A heterogeneous disorder caused by a variety of abnormalities of smooth muscle or myenteric plexus. Gastroenterology. 1983;85:538-47.

11. Kassir R, Boueil-Bourlier A, Baccot S. Jejuno-ileal diverticulitis: etiopathogenicity, diagnosis and management. Int J Surg Case Rep. 2015;10:151-3.

12. Parlakgumus A, Ezer A, Tarim A. A rare cause of gastrointestinal bleeding: jejunal diverticulosis. J Coll Physicians Surg Pak. 2016;26:870-1.

13. Coulier B, Maldague P, Bourgeois A, Broze B. Diverticula of the small bowel: CT diagnosis. Abdom Imaging. 2007;32:228-33.

14. Macari M, Faust $M$, Liang $H$, Pachter $H L$. CT of jejunal diverticulitis: imaging findings, differential diagnosis and clinical management. Clin Radiol. 2007;62:737.

15. Blam ME, Metz DC. Image of the month. A wind sock web deformity of the proximal duodenum. Gastroenterology. 2000;119:292.

16. Ross CB, Richards WO, Sharp KW, Bertram PD, Schaper PW. Diverticular disease of the jejunum and its complications. Am Surg. 1990;56:319-24.

17. Horesh N, Klang E, Gravetz A, Nevo Y, Amiel I, Amitai MM, et al. Jejunal diverticulitis. J Laparoendosc Adv Surg Tech A. 2016;26:596-9.

18. Novak JS, Tobias J, Barkin JS. Nonsurgical management of acute jejunal diverticulitis: a review. Am J Gastroenterol. 1997:92:1929-31.

19. Laiz Díez B, González González J, Ruiz-Tovar J, López Monclús J, Durán Poveda M. Jejunal pseudodiverticulosis. Three cases report. Rev Esp Enferm Dig. 2017;109:305-6.

20. Spasojevic M, Naesgaard JM, Ignjatovic D. Perforated midgut diverticulitis: revisited. World J Gastroenterol. 2012;18:4714-20.

21. Chendrasekhar A, Timberlake GA. Perforated jejunal diverticula: analysis of reported cases. Am Surg. 1995;61:984-8.

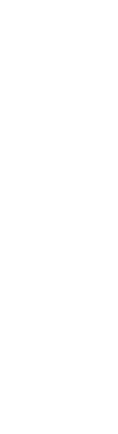

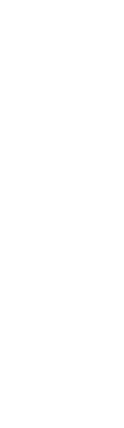

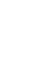

